\title{
Lifetime and Stability of Silicon Nitride Nanopores and Nanopore Arrays for Ionic Measurements
}

\author{
Yung-Chien Chou ${ }^{1}$, Paul Masih Das ${ }^{1}$, Dimitri S. Monos ${ }^{2,3}$, Marija Drndić1* \\ ${ }^{1}$ Department of Physics and Astronomy, University of Pennsylvania, Philadelphia, PA,19104, United \\ States of America \\ ${ }^{2}$ Department of Pathology and Laboratory Medicine, Perelman School of Medicine, University of \\ Pennsylvania and The Children's Hospital of Philadelphia, Philadelphia, PA, 19104, United States of \\ America \\ ${ }^{3}$ Immunogenetics Laboratory, Children's Hospital of Philadelphia, PA, 19104, United States of \\ America \\ *To whom correspondence should be addressed: drndic@physics.upenn.edu.
}

\section{Contents:}

Table S1. Measured conductance data for $1 \mathrm{M} \mathrm{KCl}$ for pore in Figure 2c.

Figure S1. TEM images and EELS analysis for a nanopore stored in $1 \mathrm{M} \mathrm{LiCl.}$

Figure S2. TEM images and phase-contrast intensity line-scan for a pore stored in $1 \mathbf{M} \mathbf{M g C l}_{2}$.

Figure S3. TEM images of pore etching in a commercial $\mathrm{SiN}_{\mathbf{x}}$ membrane in $1 \mathrm{M} \mathrm{KCl}$.

Figure S4. Dark-field (DF) TEM images of nanopore arrays and EELS analysis.

Figure S5. Variation of $\boldsymbol{d}_{T E M}$ within nanopore arrays. 


\begin{tabular}{ccc}
\hline Days & $\mathbf{G}(\mathrm{nS})$ & $\mathrm{d}_{\text {calc }}^{\mathrm{t}_{\text {eff }}=10 \mathrm{~nm} / 3 \mathrm{~nm}}$ \\
\hline 0 & 31.3 & $7.6 / 5.2$ \\
7 & 141.7 & $20.6 / 16.1$ \\
8.8 & 164.3 & $23.0 / 18.3$ \\
13.9 & 210.4 & $27.6 / 22.5$ \\
77.9 & 918.2 & $94.0 / 86.8$ \\
\hline
\end{tabular}

Table S1. Recorded conductance, G, for the nanopore stored in $1 \mathrm{M} \mathrm{KCl}$ shown in Figure 2c. Corresponding $d_{\text {calc }}$ were obtained from Eq. 1 in the main text assuming $t_{\text {eff }}=t_{\text {mem }}=10 \mathrm{~nm}$ (case 1) and $t_{\text {eff }}=t_{m e m} / 3 \approx 3 \mathrm{~nm}$ (case 2 ), respectively. The $\mathrm{I}-\mathrm{V}$ characteristics of this nanopore were consecutively measured five times, up to 78 days. 
(a)
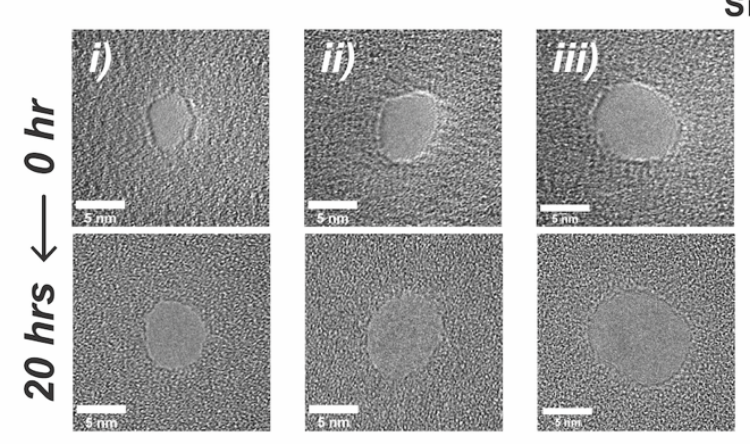

1M LiCl

$\mathrm{SiN}_{\mathrm{x}} / \mathrm{SiO}_{2} / \mathrm{Si} \mathrm{Chip}$

(b)

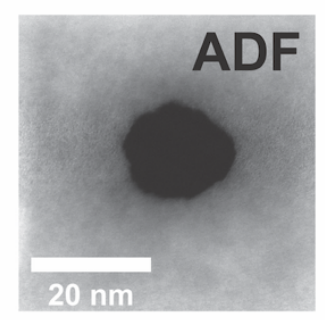

(c)
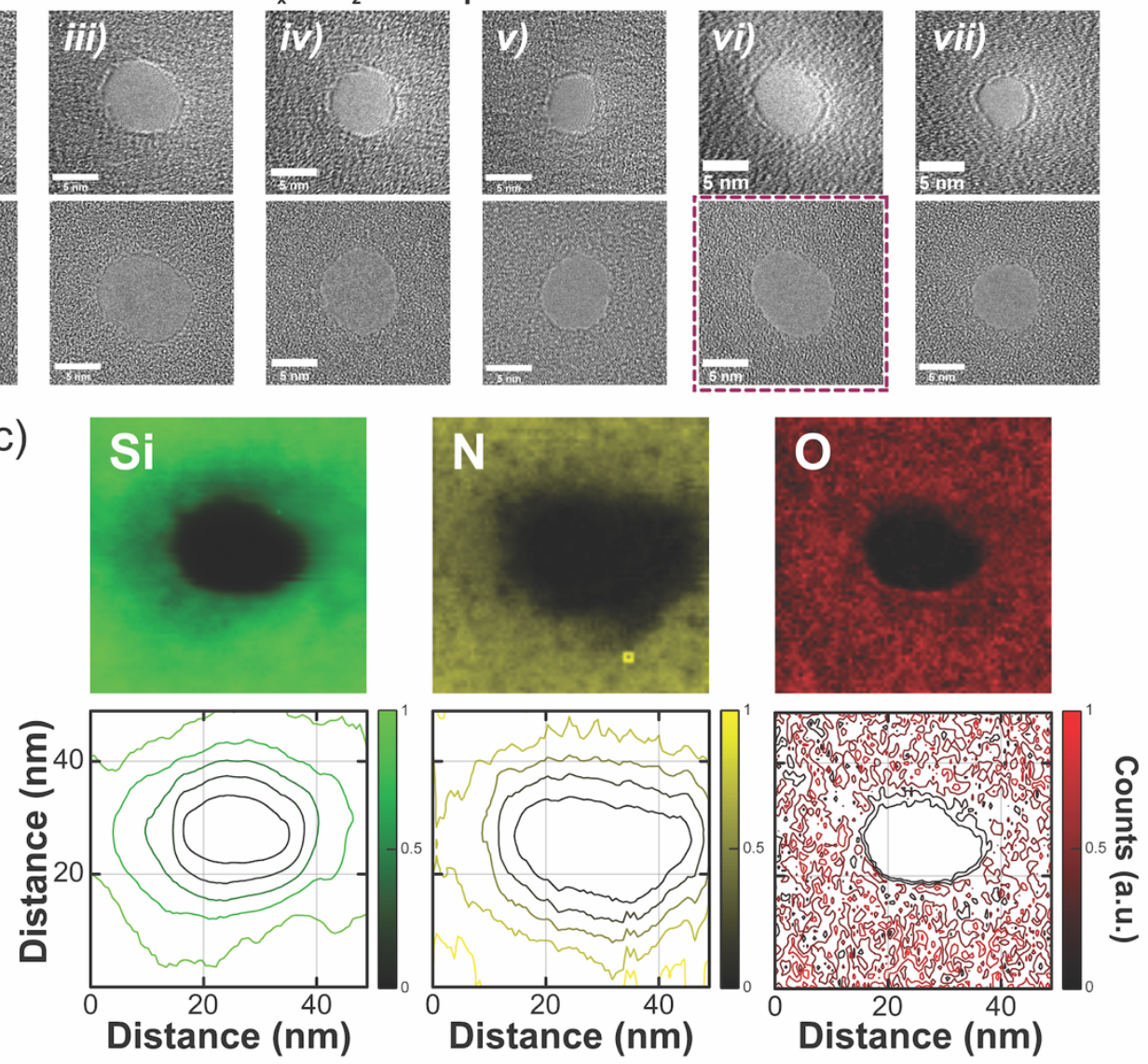

Figure S1. (a) Series of TEM images of additional seven nanopores, before and after storage in 1M LiCl for 20 hours (not shown in the main text). (b) - (c) Additional EELS 2D spatial composition map was conducted on pore (vi), marked by a purple dashed square in Figure S1 (a). Contour maps of Si $\mathrm{L}_{2,3}$ edge (green) and $\mathrm{N} \mathrm{K}$ edge (yellow) indicate that the thinned region has a diameter approximately $d_{E E L S} \approx 30 \mathrm{~nm}$. From the $\mathrm{O} \mathrm{K}$ edge (red) signals, we observe a uniformly distributed layer of native oxide. 

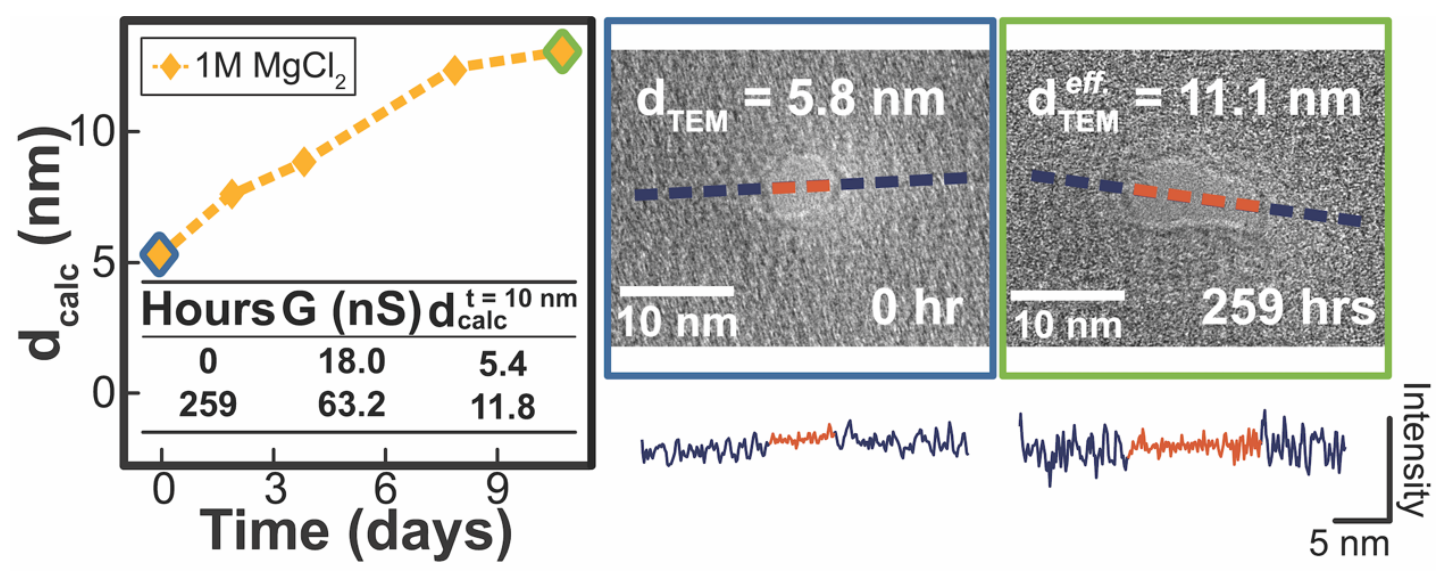

Figure S2. $d_{\text {calc }} v s$. time for a $\sim 5$-nm-diameter pore stored in $1 \mathrm{M} \mathrm{MgCl}_{2}$ over 11 days. Corresponding TEM images and TEM line scan profiles for the as-fabricated pore (blue box) and after storage in $1 \mathrm{M} \mathrm{MgCl}_{2}$ over 11 days (green box). The calculated pore diameter is listed in the inset. This pore expanded from $\sim 5 \mathrm{~nm}$ diameter to $\sim 12 \mathrm{~nm}$ diameter. Because this membrane was relatively thin $(10 \mathrm{~nm})$, there is little contrast in the TEM line scan between the membrane (blue) and the pore (red) region (there is a higher signal noise above the membrane region). 


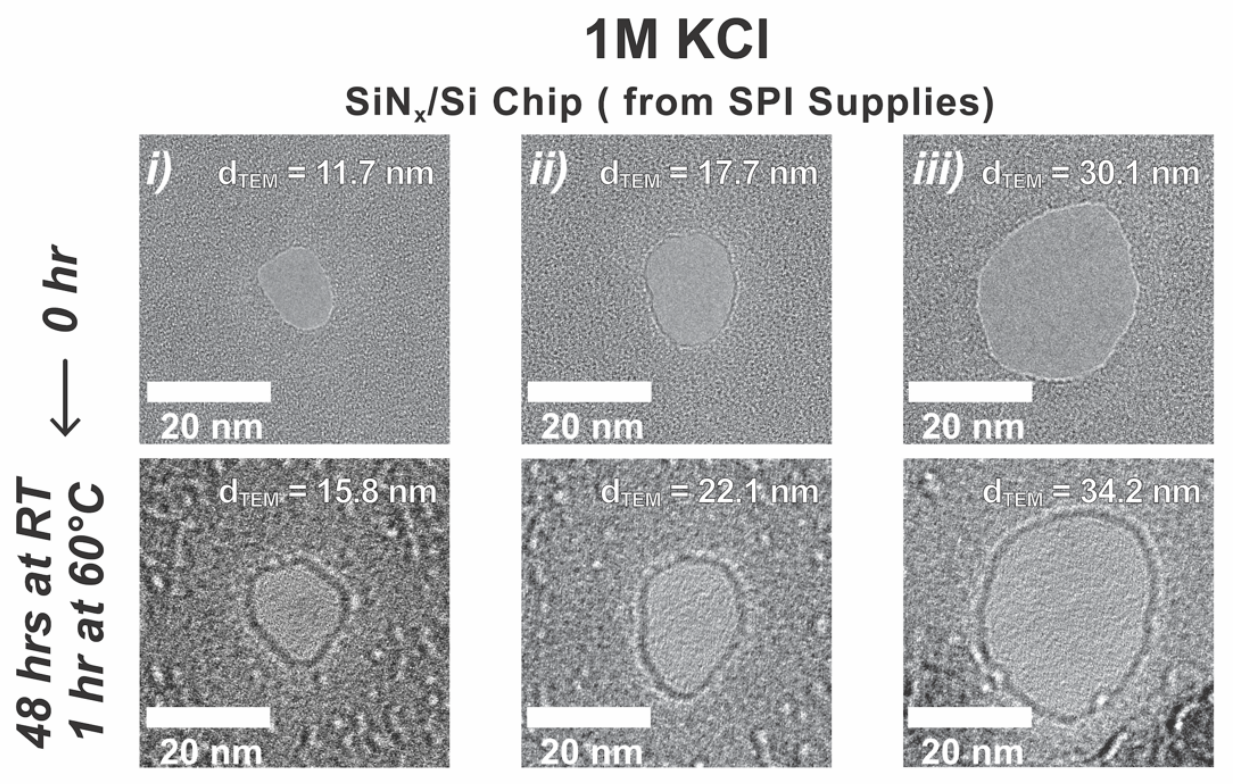

Figure S3. A commercial, 20-nm-thick silicon nitride membrane chip from SPI Supplies (Model \# 4163SN-BA) was used to fabricate nanopores via TEM drilling. A series of TEM images of three nanopores is shown, before and after storage in $1 \mathrm{M} \mathrm{KCl}$ for 48 hours at room temperature and then followed by 1 hour in the same solution heated at $60^{\circ} \mathrm{C}$. Corresponding etch rate is then calculated to be $\Delta\left(d_{T E M}\right) / \Delta t=2.1 \pm 0.1 \mathrm{~nm}$. Future measurements will investigate effects of temperature on etch rates. 
(a)
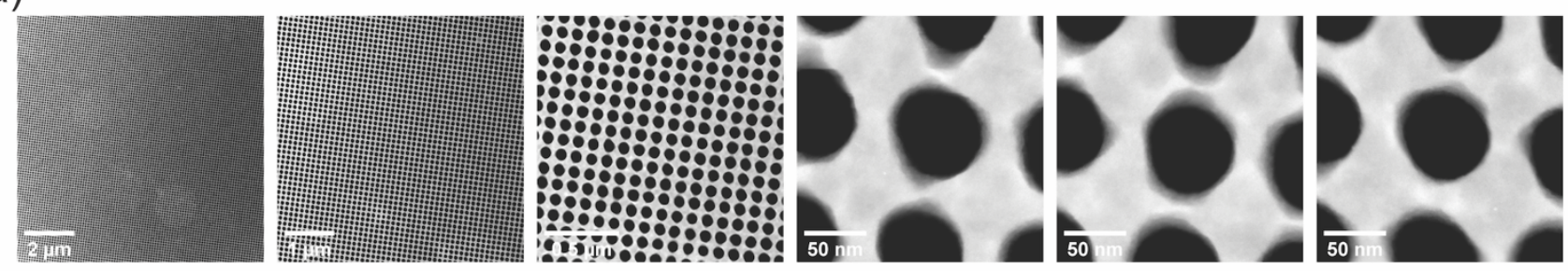

(b)

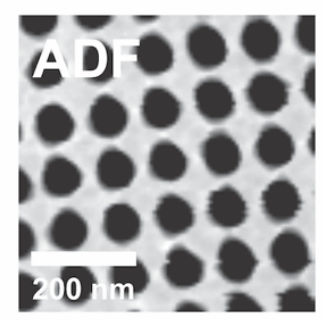

(c)
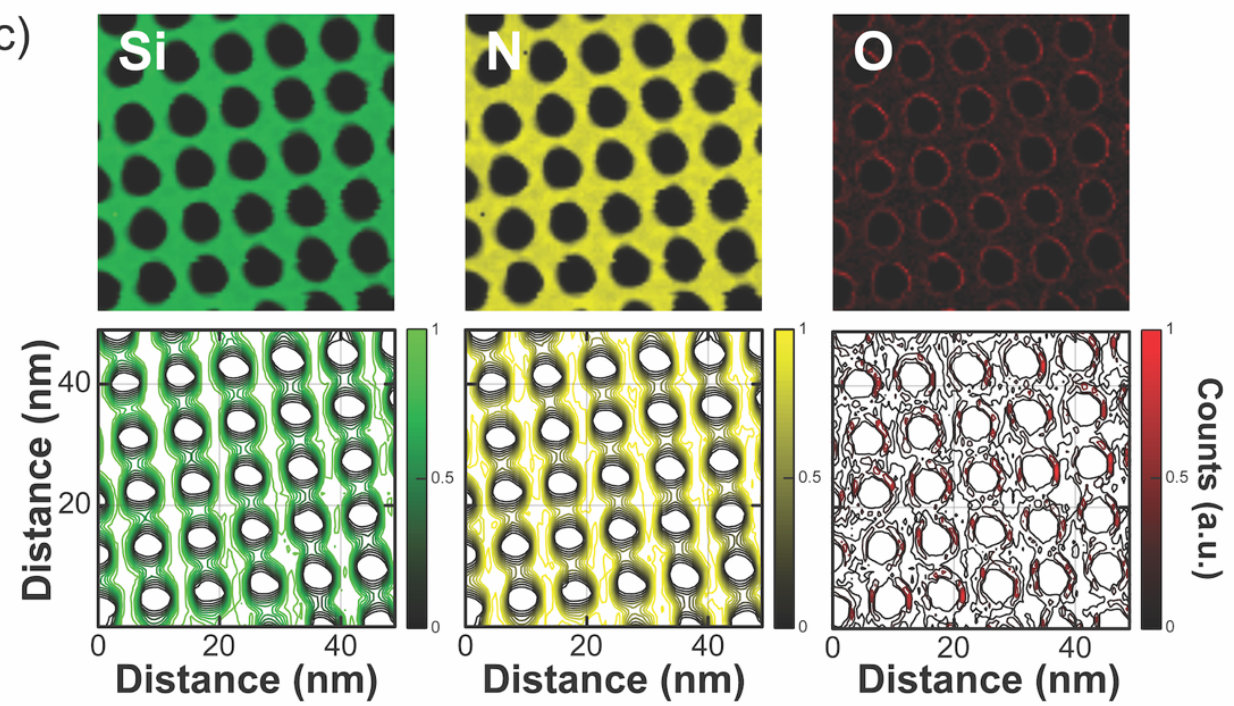

Figure S4. (a) Series of dark-field (DF) TEM images of a nanopore array after storage in 1M $\mathrm{KCl}$ for 167 hours. (b) - (c) Additional EELS 2D spatial composition maps were acquired for a region containing a 5-by-5 array of nanopores. Contour maps of $\mathrm{Si}_{2,3}$ edge (green) and $\mathrm{N} \mathrm{K}$ edge (yellow) indicate a more confined, $\mathrm{N}$-deficient region, as opposed to the case of TEM pores. An evenly lit up region of $\mathrm{O} \mathrm{K}$ edge (red) signals at the pore edge shows that a uniform layer of silicon oxide resides along the pore walls. 
(a)

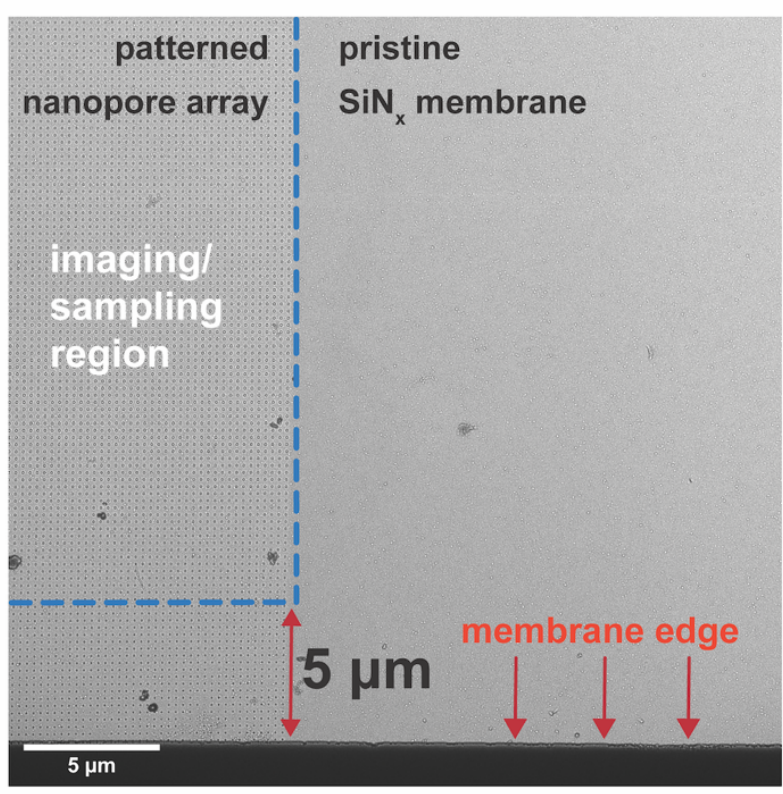

(b)

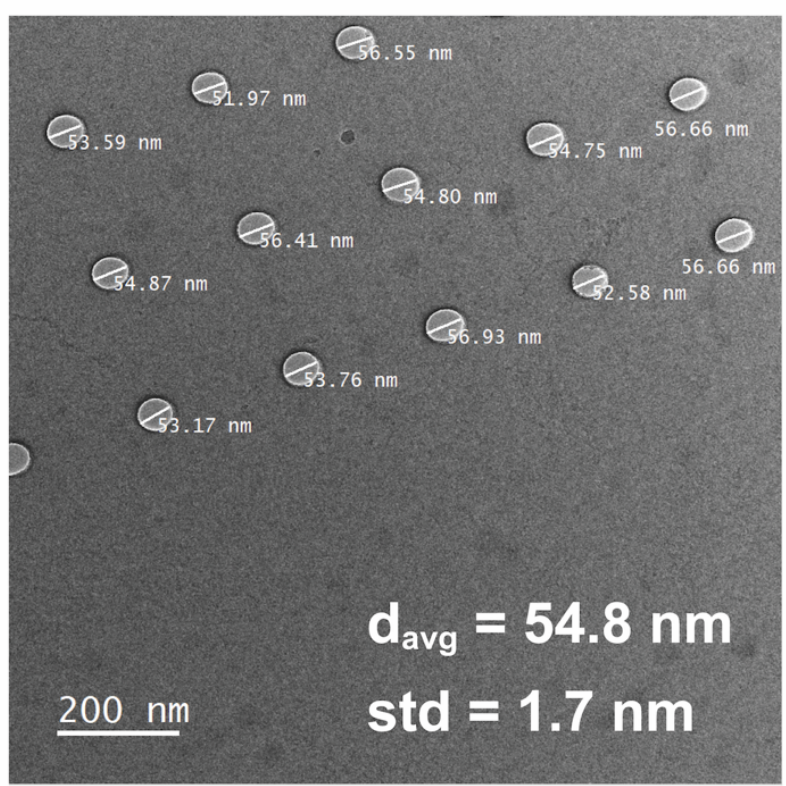

Figure S5. (a) Low-magnification TEM image of a nanopore array region. Examination and calculation of the distribution of $d_{T E M}$ in Figure 4e were randomly selected within the imaging/sampling region (enclosed by blue dashed line), which was chosen to be $5 \mu \mathrm{m}$ away from the membrane edge. (b) a high-magnification TEM image taken within imaging/sampling region was shown here. We then acquire the variation of diameters within nanopore arrays to be, $\left\langle d_{T E M}\right\rangle=54.8 \pm 1.7 \mathrm{~nm}$. 